\title{
Ground-state properties of doubly magic nuclei from the unitary-model-operator approach with the chiral two- and three-nucleon forces
}

\author{
T. Miyagi, ${ }^{1,2}$ T. Abe,${ }^{3,1}$ M. Kohno, ${ }^{4}$ P. Navrátil,,${ }^{2}$ R. Okamoto, ${ }^{5}$ T. Otsuka,${ }^{6,3,7,8}$ N. Shimizu, ${ }^{1}$ and S. R. Stroberg ${ }^{2,9}$ \\ ${ }^{1}$ Center for Nuclear Study, The University of Tokyo, 7-3-1 Hongo, Bunkyo, Tokyo 113-0033, Japan \\ ${ }^{2}$ TRIUMF, 4004 Wesbrook Mall, Vancouver, British Columbia, V6T $2 A 3$ Canada \\ ${ }^{3}$ Department of Physics, The University of Tokyo, 7-3-1, Hongo, Bunkyo, Tokyo 113-0033, Japan \\ ${ }^{4}$ Research Center for Nuclear Physics, Osaka University, Japan \\ ${ }^{5}$ Senior Academy, Kyushu Institute of Technology, Tobata, Kitakyushu 804-0015, Japan \\ ${ }^{6}$ RIKEN Nishina Center, 2-1 Hirosawa, Wako, Saitama 351-0198, Japan \\ ${ }^{7}$ National Superconducting Cyclotron Laboratory, Michigan State University, East Lansing, Michigan 48824, USA \\ ${ }^{8}$ Instituut voor Kern- en Stralingsfysica, KU Leuven, B-3001 Leuven, Belgium \\ ${ }^{9}$ Physics Department, Reed College, Portland, Oregon, 97202 USA
}

(Dated: August 23, 2021)

\begin{abstract}
The ground-state energies and radii for ${ }^{4} \mathrm{He},{ }^{16} \mathrm{O}$, and ${ }^{40} \mathrm{Ca}$ are calculated with the unitarymodel-operator approach (UMOA). In the present study, we employ the similarity renormalization group (SRG) evolved nucleon-nucleon $(N N)$ and three-nucleon $(3 N)$ interactions based on the chiral effective field theory. This is the first UMOA calculation with both $N N$ and $3 N$ interactions. The calculated ground-state energies and radii are consistent with the recent ab initio results with the same interaction. We evaluate the expectation values with two- and three-body SRG evolved radius operators, in addition to those with the bare radius operator. With the aid of the higher-body evolution of radius operator, it is seen that the calculated radii tend to be SRG resolution-scale independent. We find that the SRG evolution gives minor modifications for the radius operator.
\end{abstract}

\section{INTRODUCTION}

Recent nuclear ab initio studies are encouraged by the development, in particular, of the nuclear interactions from the chiral effective field theory $(\chi \mathrm{EFT})[1,2]$. In the $\chi \mathrm{EFT}$, nuclear interactions are obtained through the perturbation expansion of the chiral Lagrangian which is the effective Lagrangian of the quantum chromodynamics. By taking into account the higher-order expansion terms, the systematic improvement of the nuclear interactions can be expected, for recent example, see Refs. [3-5]. As another advantage of employing the $\chi \mathrm{EFT}$, the consideration of higer-order terms in the perturbation series allows us the systematic derivation of the three-nucleon $(3 N)$ interaction. With the development of the $\chi$ EFT interactions, the impacts of the $3 N$ force on nuclear structure calculation have been discussed extensively, for example, in light nuclei [6-9], medium-mass nuclei [10-18], and infinite nuclear matter [19-22].

Besides the progress in nuclear forces, the advancement in many-body method is also necessary. To deal with nuclear many-body problems, one can use the $a b$ initio calculation methods such as no-core shell model (NCSM) [23], quantum Monte Carlo methods [24], nuclear lattice EFT calculations [25], coupledcluster method [26], self-consistent Green's function method [27], in-medium similarity renormalization group approach [28], and many-body perturbation theory [29, 30]. Over the past decade, the tremendous advancements were made in nuclear ab initio studies. Nowadays, the capability of the $a b$ initio calculations has reached mass region $A \sim 100[18,29,31]$. In addition to these methods, we can apply the unitary-model-operator approach (UMOA) $[32,33]$ to solve the nuclear many-body
Schrödinger equation. In the UMOA, a unitary transformation of the Hamiltonian is constructed so that the the one-particle-one-hole and two-particle-two-hole excitations are limitated from the transformed Hamiltonian. So far, we calculated the ground-state energies and radii for some closed shell nuclei with only the nucleon-nucleon $(N N)$ interactions. In this work, we include the $3 N$ interaction effect to the UMOA calculation for the first time.

Due to the non-perturbative nature of the nuclear force, in most cases, it is not possible to apply directly the nuclear interactions to the many-body calculations. To bridge the gap between nuclear forces and manybody calculations, we evolve the nuclear Hamiltonian with the similarity renormalization group (SRG) flow equation [34]. Through the SRG evolution, we obtain the Hamiltonian whose coupling between low- and highmomentum regions is suppressed. With such nuclear interactions, recent $a b$ initio results significantly underestimate the nuclear radii, see for instance Refs. [16, 17, 28, 35]. Since the nuclear size can affect the single-particle level structure of a nucleus, the reproduction of nuclear radii is one of the fundamental issues to discuss the nuclear structure. As seen in NCSM calculations for fewbody systems [36, 37], we should also evolve consistently other operators than the Hamiltonian. In this work, we demonstrate the effect of the SRG evolution on radius operator.

This paper is organized as follows. The Hamiltonian and radius operators employed in this work are introduced in Sec. II. Section III describes briefly the formalism of the UMOA. In Sec. IV, the numerical results for ${ }^{4} \mathrm{He},{ }^{16} \mathrm{O}$, and ${ }^{40} \mathrm{Ca}$ are given. After comfirming convergence and consistency with the other $a b$ initio results, 
the effects of the SRG evolution on radius operator are discussed. The summary of the present work is given in Sec. V.

\section{HAMILTONIAN AND RADIUS OPERATORS}

Our starting Hamiltonian is composed of the kinetic, $N N$, and $3 N$ terms:

$$
H=T+V^{N N}+V^{3 N} .
$$

Here, $T$ is the kinetic energy operator. The $V^{N N}$ and $V^{3 N}$ indicate the $N N$ and $3 N$ interactions, respectively. Usually, the bare Hamiltonian $H$ is too "hard" to apply for many-body calculation methods. It causes the slow convergence with respect to the size of model space and calculations demand the huge amount of computational resources. To obtain the converged results from the feasible model-space calculations, the similarityrenormalization group (SRG) evolution [34] is employed in this work. We consider the unitary transformation of the original Hamiltonian:

$$
H(\alpha)=U_{\mathrm{SRG}}^{\dagger}(\alpha) H U_{\mathrm{SRG}}(\alpha) .
$$

Here, $U_{\mathrm{SRG}}(\alpha)$ is the unitary transformation operator and is evolved by the flow equation:

$$
\frac{d U_{\mathrm{SRG}}(\alpha)}{d \alpha}=U_{\mathrm{SRG}}(\alpha) \eta(\alpha) .
$$

The $\alpha$ is the resolution scale parameter of the flow equation in unit of $\mathrm{fm}^{4}$. The $\eta$ is called the generator of the SRG evolution and is taken as $\eta(\alpha)=[T, H(\alpha)]$. Note that the initial condition for $U_{\mathrm{SRG}}(\alpha)$ is $U_{\mathrm{SRG}}(0)=\mathbb{1}$. Alternative to $\alpha$, it is common to use $\lambda_{\mathrm{SRG}}=\alpha^{-1 / 4}$ for controlling the flow equation Eq. (3). The Hamiltonian is transformed by Eq. (2) from $\lambda_{\mathrm{SRG}}=\infty \mathrm{fm}^{-1}$ to lower values where the interaction is "soft" enough for convergence of the many-body calculation methods. As discussed, for example in Ref. [38], the SRG evolution, however, induces the many-body forces:

$$
H\left(\lambda_{\mathrm{SRG}}\right)=T+V^{N N}\left(\lambda_{\mathrm{SRG}}\right)+V^{3 N}\left(\lambda_{\mathrm{SRG}}\right)+\cdots .
$$

Consequently, during the SRG evolution, we should keep many-body terms, as many as possible, even if the starting Hamiltonian does not include the many-body interactions. In this work, three types of Hamiltonians are used. First one, labeled by " $N N$-only", is obtained by keeping only the $N N$ interaction during the SRG evolution starting without the genuine $3 N$ interaction. Second one " $N N+3 N$-ind" is obtained by keeping the $N N$ and $3 N$ interactions during the SRG evolution starting without the genuine $3 N$ interaction. Third one " $N N+3 N-$ full" is obtained by keeping the $N N$ and $3 N$ interactions during the SRG evolution starting with the genuine $3 N$ interaction.
To evaluate nuclear root-mean-squared radii, we should transform the radius operator in the same manner as the Hamiltonian:

$$
r^{2}(\alpha)=U_{\mathrm{SRG}}^{\dagger}(\alpha) r^{2} U_{\mathrm{SRG}}(\alpha) .
$$

The original radius operator is defined as

$$
r^{2}=r^{2(2)}=\frac{1}{A^{2}} \sum_{i<j}\left(\mathbf{r}_{i}-\mathbf{r}_{j}\right)^{2}
$$

with the coordinate vector of the $i$-th nucleon $\mathbf{r}_{i}$ and number of nucleon $A$. In the same manner as the Hamiltonian, the many-body radius operator can be induced through the SRG evolution:

$$
r^{2}\left(\lambda_{\mathrm{SRG}}\right)=r^{2(2)}\left(\lambda_{\mathrm{SRG}}\right)+r^{2(3)}\left(\lambda_{\mathrm{SRG}}\right)+\cdots .
$$

Following to Refs. $[36,37]$, we keep up to three-body terms.

To perform many-body calculations, it is numerically efficient to transform to the laboratory frame. Then, our Hamiltonian and radius operators can be rewritten as

$$
\begin{aligned}
& H=T^{(1)}+\left[T^{(2)}+V^{N N}\left(\lambda_{\mathrm{SRG}}\right)\right]+V^{3 N}\left(\lambda_{\mathrm{SRG}}\right), \\
& r^{2}=r^{2(1)}+\left[r^{2(2)}\left(\lambda_{\mathrm{SRG}}\right)-r^{2(1)}\right]+r^{2(3)}\left(\lambda_{\mathrm{SRG}}\right) .
\end{aligned}
$$

Here, we use $T^{(1)}=\sum_{i}(1-1 / A) \mathbf{p}_{i}^{2} / 2 m$ with the $i_{-}$ th nucleon momentum $\mathbf{p}_{i}$ and nucleon mass $m, T^{(2)}=$ $-\sum_{i<j} \mathbf{p}_{i} \cdot \mathbf{p}_{j} / m A$, and $r^{2(1)}=(1-1 / A) \sum_{i} \mathbf{r}_{i}^{2}$. Note that $r^{2(1)}$ is chosen so that $r^{2(2)}\left(\lambda_{\mathrm{SRG}}\right)-r^{2(1)}$ goes $-\sum_{i<j} \mathbf{r}_{i} \cdot \mathbf{r}_{j} / A$ in the limit of $\lambda_{\mathrm{SRG}} \rightarrow \infty$. In the second quantization form, they are

$$
\begin{aligned}
H & =\sum_{a_{1} a_{2}} t_{a_{1} a_{2}} c_{a_{1}}^{\dagger} c_{a_{2}} \\
& +\left(\frac{1}{2 !}\right)^{2} \sum_{a_{1} a_{2} a_{3} a_{4}} v_{a_{1} a_{2} a_{3} a_{4}}^{(2)} c_{a_{1}}^{\dagger} c_{a_{2}}^{\dagger} c_{a_{4}} c_{a_{3}} \\
& +\left(\frac{1}{3 !}\right)^{2} \sum_{a_{1} a_{2} a_{3} a_{4} a_{5} a_{6}} v_{a_{1} a_{2} a_{3} a_{4} a_{5} a_{6}}^{(3)} c_{a_{1}}^{\dagger} c_{a_{2}}^{\dagger} c_{a_{3}}^{\dagger} c_{a_{6}} c_{a_{5}} c_{a_{4}}, \\
r^{2} & =\sum_{a_{1} a_{2}} r_{a_{1} a_{2}}^{2(1)} c_{a_{1}}^{\dagger} c_{a_{2}} \\
& +\left(\frac{1}{2 !}\right)^{2} \sum_{a_{1} a_{2} a_{3} a_{4}} r_{a_{1} a_{2} a_{3} a_{4}}^{2(2)} c_{a_{1}}^{\dagger} c_{a_{2}}^{\dagger} c_{a_{4}} c_{a_{3}} \\
& +\left(\frac{1}{3 !}\right)^{2} \sum_{a_{1} a_{2} a_{3} a_{4} a_{5} a_{6}} r_{a_{1} a_{2} a_{3} a_{4} a_{5} a_{6}}^{2(3)} c_{a_{1}}^{\dagger} c_{a_{2}}^{\dagger} c_{a_{3}}^{\dagger} c_{a_{6}} c_{a_{5}} c_{a_{4}} .
\end{aligned}
$$

Here, $c_{a}\left(c_{a}^{\dagger}\right)$ is the annihilation (creation) operator of the nucleon at the state $a$. In Eqs. (10) and (11), shorthand 
notations,

$$
\begin{aligned}
t_{a_{1} a_{2}} & =\left\langle a_{1}\left|T^{(1)}\right| a_{2}\right\rangle \\
v_{a_{1} a_{2} a_{3} a_{4}}^{(2)} & =\left\langle a_{1} a_{2}\left|V^{N N}\left(\lambda_{\mathrm{SRG}}\right)+T^{(2)}\right| a_{3} a_{4}\right\rangle \\
v_{a_{1} a_{2} a_{3} a_{4} a_{5} a_{6}}^{(3)} & =\left\langle a_{1} a_{2} a_{3}\left|V^{3 N}\left(\lambda_{\mathrm{SRG}}\right)\right| a_{4} a_{5} a_{6}\right\rangle \\
r_{a_{1} a_{2}}^{2(1)} & =\left\langle a_{1}\left|r^{2(1)}\right| a_{2}\right\rangle \\
r_{a_{1} a_{2} a_{3} a_{4}}^{2(2)} & =\left\langle a_{1} a_{2}\left|r^{2(2)}\left(\lambda_{\mathrm{SRG}}\right)-\frac{1}{A-1} r^{2(1)}\right| a_{3} a_{4}\right\rangle,
\end{aligned}
$$$$
r_{a_{1} a_{2} a_{3} a_{4} a_{5} a_{6}}^{2(3)}=\left\langle a_{1} a_{2} a_{3}\left|r^{2(3)}\left(\lambda_{\mathrm{SRG}}\right)\right| a_{4} a_{5} a_{6}\right\rangle,
$$

are used for one-body-kinetic-term, two-bodyinteraction, three-body-interaction, one-body-radius, two-body-radius, and three-body-radius matrix elements, respectively. The factor $1 /(A-1)$ in two-bodyradius matrix element is due to the normalization when a one-body operator is used as a two-body operator. Because of the computational limitation, however, the direct treatment of the three-body matrix elements is still challenging. Therefore, we follow the recent nuclear $a b$ initio studies and introduce the normal-ordered two-body (NO2B) approximation [39, 40]. The key of the approximation is a rearrangement of the three-body term with respect to a reference state $|\Phi\rangle$. After the rearrangement, the zero-, one-, two-, and three-body pieces show up. In the NO2B approximation, the residual three-body piece is discarded. To apply to the UMOA framework, we take normal order again with respect to the nucleon vacuum state. Then, the Hamiltonian is

$$
\begin{aligned}
H & \approx h^{(0), \mathrm{NO} 2 \mathrm{~B}}+\sum_{a_{1} a_{2}} h_{a_{1} a_{2}}^{(1), \mathrm{NO} 2 \mathrm{~B}} c_{a_{1}}^{\dagger} c_{a_{2}} \\
& +\left(\frac{1}{2 !}\right)^{2} \sum_{a_{1} a_{2} a_{3} a_{4}} h_{a_{1} a_{2} a_{3} a_{4}}^{(2), \mathrm{NO} 2 \mathrm{~B}} c_{a_{1}}^{\dagger} c_{a_{2}}^{\dagger} c_{a_{4}} c_{a_{3}}
\end{aligned}
$$

with

$$
\begin{aligned}
& h^{(0), \mathrm{NO} 2 \mathrm{~B}}=\frac{1}{6} \sum_{a b c} v_{a b c a b c}^{(3)} n_{a} n_{b} n_{c}, \\
& h_{a_{1} a_{2}}^{(1), \mathrm{NO} 2 \mathrm{~B}}=t_{a_{1} a_{2}}-\frac{1}{2} \sum_{b c} v_{a_{1} b c a_{2} b c}^{(3)} n_{b} n_{c}, \\
& h_{a_{1} a_{2} a_{3} a_{4}}^{(2), \mathrm{NO} 2 \mathrm{~B}}=v_{a_{1} a_{2} a_{3} a_{4}}^{(2)}+\sum_{b} v_{a_{1} a_{2} b a_{3} a_{4} b}^{(3)} n_{b} .
\end{aligned}
$$

Here, $n_{a}$ is an occupation number for the orbit $a$, i.e. $n_{a}=1\left(n_{a}=0\right)$ where $a$ is below (above) the Fermi level. To minimize the effect of the truncated residual three-body piece, the choice of $|\Phi\rangle$ is crucial. In this work, we use the Hartree-Fock state as $|\Phi\rangle$. Same as the Hamiltonian, we employ the NO2B approximated radius operator:

$$
\begin{aligned}
r^{2} & \approx r^{2(0), \mathrm{NO} 2 \mathrm{~B}}+\sum_{a_{1} a_{2}} r_{a_{1} a_{2}}^{2(1), \mathrm{NO} 2 \mathrm{~B}} c_{a_{1}}^{\dagger} c_{a_{2}} \\
& +\left(\frac{1}{2 !}\right)^{2} \sum_{a_{1} a_{2} a_{3} a_{4}} r_{a_{1} a_{2} a_{3} a_{4}}^{2(2), \mathrm{NO} 2 \mathrm{~B}} c_{a_{1}}^{\dagger} c_{a_{2}}^{\dagger} c_{a_{4}} c_{a_{3}} .
\end{aligned}
$$

with

$$
\begin{aligned}
& r^{2(0), \mathrm{NO} 2 \mathrm{~B}}=\frac{1}{6} \sum_{a b c} r_{a b c a b c}^{2(3)} n_{a} n_{b} n_{c}, \\
& r_{a_{1} a_{2}}^{2(1), \mathrm{NO} 2 \mathrm{~B}}=r_{a_{1} a_{2}}^{2(1)}-\frac{1}{2} \sum_{b c} r_{a_{1} b c a_{2} b c}^{2(3)} n_{b} n_{c} \\
& r_{a_{1} a_{2} a_{3} a_{4}}^{2(2), \mathrm{NO} 2 \mathrm{~B}}=r_{a_{1} a_{2} a_{3} a_{4}}^{2(2)}+\sum_{b} r_{a_{1} a_{2} b a_{3} a_{4} b}^{2(3)} n_{b} .
\end{aligned}
$$

\section{UNITARY-MODEL-OPERATOR APPROACH}

To solve the many-body Schrödinger equation associated with the Hamiltonian Eq. (18), the UMOA [32, 33, $42,43]$ is employed in this work. In the UMOA, we construct the effective Hamiltonian with the unitary transformation:

$$
\widetilde{H}=U^{\dagger} H U
$$

The $U$ is defined by the product of two exponential operators,

$$
U=e^{S^{(1)}} e^{S^{(2)}}
$$

where $S^{(1)}$ and $S^{(2)}$ are anti-hermitian one- and two-body correlation operators, respectively. Note that the sole use of $S^{(1)}\left(S^{(2)}=0\right)$ reduces the UMOA to the HartreeFock (HF) theory. The $S^{(1)}$ and $S^{(2)}$ are specified by applying iteratively the Okubo-Lee-Suzuki method [4446] so that $\widetilde{H}$ does not induce the one-particle-one-hole and two-particle-two-hole excitations from the reference state $|\Phi\rangle$. Since the unitary transformation (26) can induce many-body interactions, $\widetilde{H}$ can include many-body operators even if the original Hamiltonian is restricted up to the two-body interaction. In actual calculations, we decompose $\widetilde{H}$ with the cluster expansion and truncate the effect of the four- and higher-body cluster terms:

$$
\begin{aligned}
\widetilde{H} & \approx \sum_{a_{1} a_{2}} \widetilde{H}_{a_{1} a_{2}}^{(1)} c_{a_{1}}^{\dagger} c_{a_{2}} \\
& +\frac{1}{4} \sum_{a_{1} a_{2} a_{3} a_{4}} \widetilde{H}_{a_{1} a_{2} a_{3} a_{4}}^{(2)} c_{a_{1}}^{\dagger} c_{a_{2}}^{\dagger} c_{a_{4}} c_{a_{3}} \\
& +\frac{1}{36} \sum_{a_{1} a_{2} a_{3} a_{4} a_{5} a_{6}} \widetilde{H}_{a_{1} a_{2} a_{3} a_{4} a_{5} a_{6}}^{(3)} c_{a_{1}}^{\dagger} c_{a_{2}}^{\dagger} c_{a_{3}}^{\dagger} c_{a_{6}} c_{a_{5}} c_{a_{4}},
\end{aligned}
$$

where $\widetilde{H}_{a_{1} a_{2}}^{(1)}, \widetilde{H}_{a_{1} a_{2} a_{3} a_{4}}^{(2)}$, and $\widetilde{H}_{a_{1} a_{2} a_{3} a_{4} a_{5} a_{6}}^{(3)}$ are the one-, two-, and three-body matrix elements, respectively (see, for example, Ref. [33] for more details). Then, the ground-state energy $E_{\text {g.s. }}$ can be obtained approximately 
TABLE I. Hugenholtz diagrams for the ground-state energy up to the third order. Note that the first order contributions are omitted. The cross and dot indicate the one- and twobody part of Hamiltonian, respectively. The diagram rules are same as in Ref. [41].

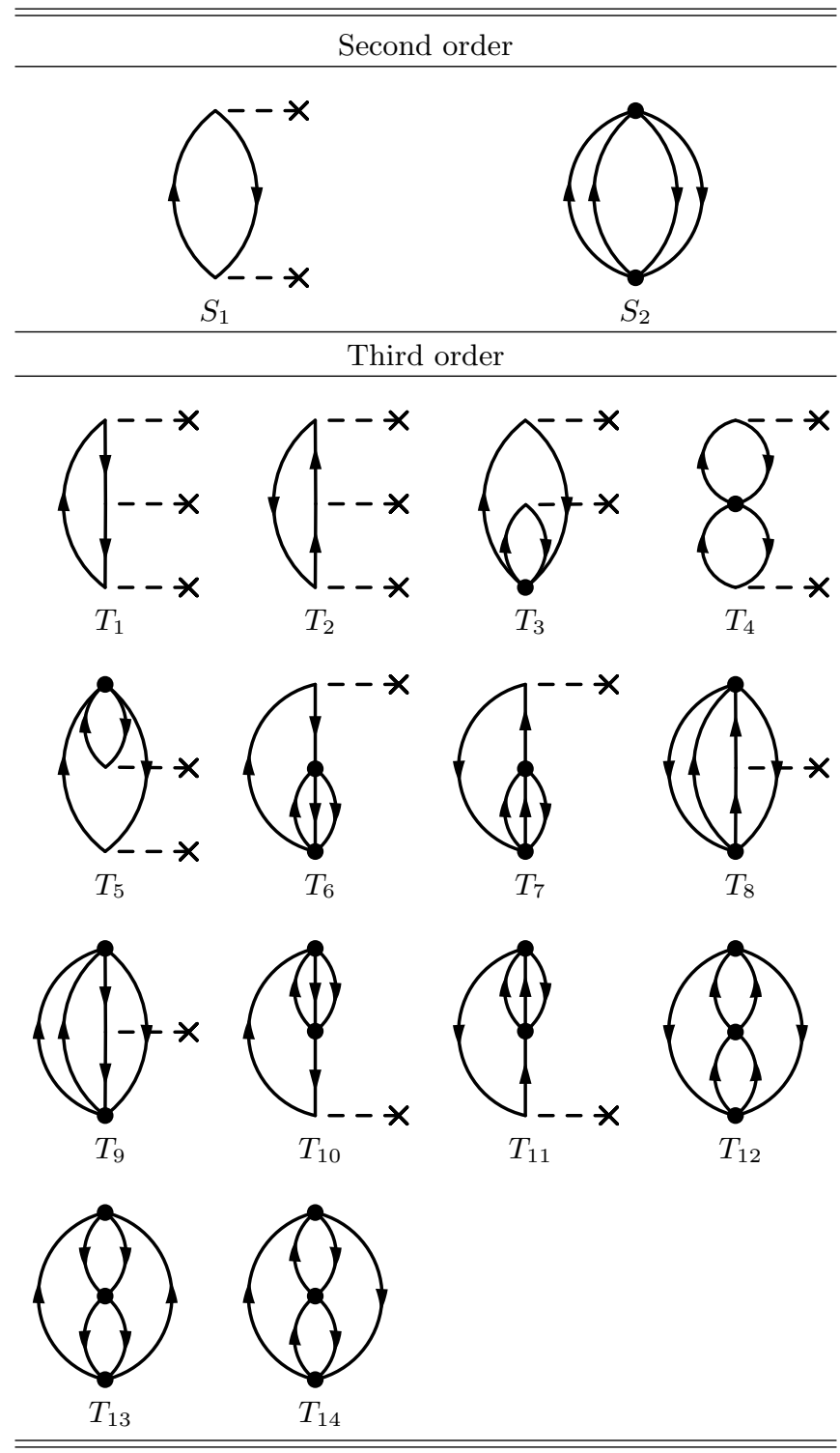

by

$$
\begin{aligned}
E_{\mathrm{g} . \mathrm{s} .} & \approx E^{1,2 \mathrm{BC}}+E^{3 \mathrm{BC}} \\
E^{1,2 \mathrm{BC}} & =\sum_{a} \widetilde{H}_{a a}^{(1)} n_{a}+\frac{1}{2} \sum_{a b} \widetilde{H}_{a b a b}^{(2)} n_{a} n_{b}, \\
E^{3 \mathrm{BC}} & =\frac{1}{6} \sum_{a b c} \widetilde{H}_{a b c a b c}^{(3)} n_{a} n_{b} n_{c} .
\end{aligned}
$$

Since the direct treatment of three-body term demands huge computational resources, however, the contribution of three-body cluster term is approximately evaluated up to second order of $S^{(2)}[32]$ :

$$
\begin{aligned}
E^{3 \mathrm{BC}} & \approx \frac{1}{4} \sum_{a b c d} \sum_{e f} \widetilde{H}_{a b c d}^{(2)} S_{e f a b}^{(2)} S_{e f c d}^{(2)} n_{a} n_{b} n_{c} n_{d} \bar{n}_{e} \bar{n}_{f} \\
& +\sum_{a b c} \sum_{d e f} \widetilde{H}_{a d c f}^{(2)} S_{d e b c}^{(2)} S_{e f a b}^{(2)} n_{a} n_{b} n_{c} \bar{n}_{d} \bar{n}_{e} \bar{n}_{f} .
\end{aligned}
$$

The $S_{a b c d}^{(2)}$ is the matrix element of the two-body correlation operator and $\bar{n}_{a}=1-n_{a}$ is used. To clarify the contribution of each cluster term, the comparison with the many-body perturbation theory (MBPT) would be useful. Table I shows the diagrams for the ground-state energy from the third-order MBPT. Following to the perturbative derivation of correlation operators, shown for example in Refs [47, 48], the contribution of each cluster term to the ground-state energy can be derived. In terms of the many-body perturbation theory, $E^{1,2 \mathrm{BC}}, E^{3 \mathrm{BC}}$, and $E_{\text {g.s. }}$ are

$$
\begin{aligned}
E^{1,2 \mathrm{BC}}=E_{1}+\sum_{i=1}^{2} S_{i}+\sum_{i=1}^{12} T_{i}-T_{13} & +(\text { higher order terms }) \\
E^{3 \mathrm{BC}}=2 T_{13}+ & T_{14}+(\text { higher order terms }) \\
E_{\text {g.s. }}=E_{1}+ & \sum_{i}^{2} S_{i}+\sum_{i=1}^{14} T_{i} \\
& +(\text { higher order terms }) .
\end{aligned}
$$

Here, $S_{i}$ and $T_{i}$ are the second- and third-order contributions shown in Table I, respectively, and $E_{1}$ is the first order ground-state energy. At one-plus-two-body cluster level, the third-order diagrams are not completed. The three-body cluster term contributions compensate the third order [49]. Note that $S_{1}$ and $T_{1}$ to $T_{11}$ vanishes when the HF basis is employed.

To evaluate the expectation value of the radius operator obtained in Eq. (22), the effective operator $\widetilde{r}^{2}$ is used:

$$
\widetilde{r}^{2}=U^{\dagger} r^{2} U
$$

Similarly to the Hamiltonian, the unitary transformation of the radius operator induces the many-body terms. However, results examined here are calculated keeping up to two-body terms and does not include any contributions from three- and higher-body terms [33]:

$$
\begin{aligned}
\widetilde{r}^{2} & \approx \sum_{a_{1} a_{2}} \widetilde{r}_{a_{1} a_{2}}^{2(1)} c_{a_{1}}^{\dagger} c_{a_{2}} \\
& +\frac{1}{4} \sum_{a_{1} a_{2} a_{3} a_{4}} \widetilde{r}_{a_{1} a_{2} a_{3} a_{4}}^{2(2)} c_{a_{1}}^{\dagger} c_{a_{2}}^{\dagger} c_{a_{4}} c_{a_{3}} .
\end{aligned}
$$

Then, the mean-squared radius $r_{\text {g.s. }}^{2}$ is approximately evaluated as

$$
r_{\mathrm{g} . \mathrm{s} .}^{2} \approx \sum_{a} \widetilde{r}_{a a}^{2(1)} n_{a}+\frac{1}{2} \sum_{a b} \widetilde{r}_{a b a b}^{2(2)} n_{a} n_{b} .
$$




\section{RESULTS AND DISCUSSIONS}

In this work, we use the next-to-next-to-next-to leading order $\left(\mathrm{N}^{3} \mathrm{LO}\right) N N$ interaction by Entem and Machleidt [51] and local form $\mathrm{N}^{2} \mathrm{LO} 3 N$ interaction [52] from $\chi$ EFT. Both two- and three-body SRG evolutions are done in the harmonic-oscillator (HO) space. The twobody interactions are obtained from the $N_{\max }=200$ space calculations. Here, $N_{\max }$ is the boundary of the $\mathrm{HO}$ quantum number for the two-body relative coordinate and is $N_{\max }=\max (2 n+l)$ with the radial quantum number $n$ and angular momentum $l$. Following Ref. [53], the three-body SRG evolution is done in ramp A model space defined in Fig. 3 in Ref [53]. To obtain the three-body matrix element, the frequency conversion technique [53] is used with the parent $\mathrm{HO}$ energy $\hbar \omega=35 \mathrm{MeV}$ matrix elements. For $\mathrm{N}^{2} \mathrm{LO} 3 \mathrm{~N}$ interaction, we use $c_{D}=-0.2$, $c_{E}=0.098$, and $\Lambda_{3 N}=400 \mathrm{MeV} / c$ [39], so as to compare with the other $a b$ initio calculation results. Note that the low-energy constant $c_{D}$ used here does not fit the ${ }^{3} \mathrm{H}$ half-life as claimed in the past $[54,55]$. The impact of the modification of the $3 N$ force with the $c_{D}$ that fits ${ }^{3} \mathrm{H}$ half-life will be discussed in the forthcoming publications. The size of the contributions from induced many-body forces can be estimated from the SRG resolution scale, $\lambda_{\mathrm{SRG}}$, dependence of calculated results. To do so, we employ three SRG resolution scales $\lambda_{\mathrm{SRG}}=1.88,2.0$, and $2.24 \mathrm{fm}^{-1}$. The NO2B approximated Hamiltonian is obtained through the $\mathrm{HF}$ calculations at $e_{3 \max }=14$. Here, $e_{3 \text { max }}$ is introduced to handle the three-body matrix element and is $e_{3 \max }=\max \left(2 n_{1}+l_{1}+2 n_{2}+l_{2}+2 n_{3}+l_{3}\right)$ with the single-particle radial quantum number $n_{i}(i=1,2,3)$ and angular momentum $l_{i}(i=1,2,3)$. We checked that the changing from $e_{3 \max }=12$ to $e_{3 \max }=14$ affects less than $1 \%$ of total ground-state energies for nuclei calculated in the present work. UMOA calculations are done in the model space defined by $e_{\max }=\max \left(2 n_{1}+l_{1}\right)$ [33].

\section{A. Ground-state energies}

Figure 1 shows the convergence property of the groundstate energies for ${ }^{4} \mathrm{He},{ }^{16} \mathrm{O}$, and ${ }^{40} \mathrm{Ca}$ calculated with the $N N+3 N$-full interaction from $\chi \mathrm{EFT}$ evolved up to $\lambda_{\mathrm{SRG}}=1.88 \mathrm{fm}^{-1}$. Our calculations are done with varying $\hbar \omega$ and $e_{\max }$ to see the numerical convergence. Note that the final results should not depend on $\hbar \omega$ because the initial Hamiltonian Eq. (1) does not include $\hbar \omega$. Similar to other ab initio calculations, our groundstate energies show parabolic $\hbar \omega$-dependence at small $e_{\max }$ and gain with increasing $e_{\max }$. For all cases examined here, $\hbar \omega$ - and $e_{\max }$-independent results are obtained $e_{\max }=14$. The results with $e_{\max }=14$ and $\hbar \omega=25 \mathrm{MeV}$ are used in the following discussion.

To investigate the contributions of the cluster expansion, in Fig. 2, it is illustrated that the comparison among UMOA, Hartree-Fock basis many-body perturbation theory (HF-MBPT), and coupled-cluster method (CCM)
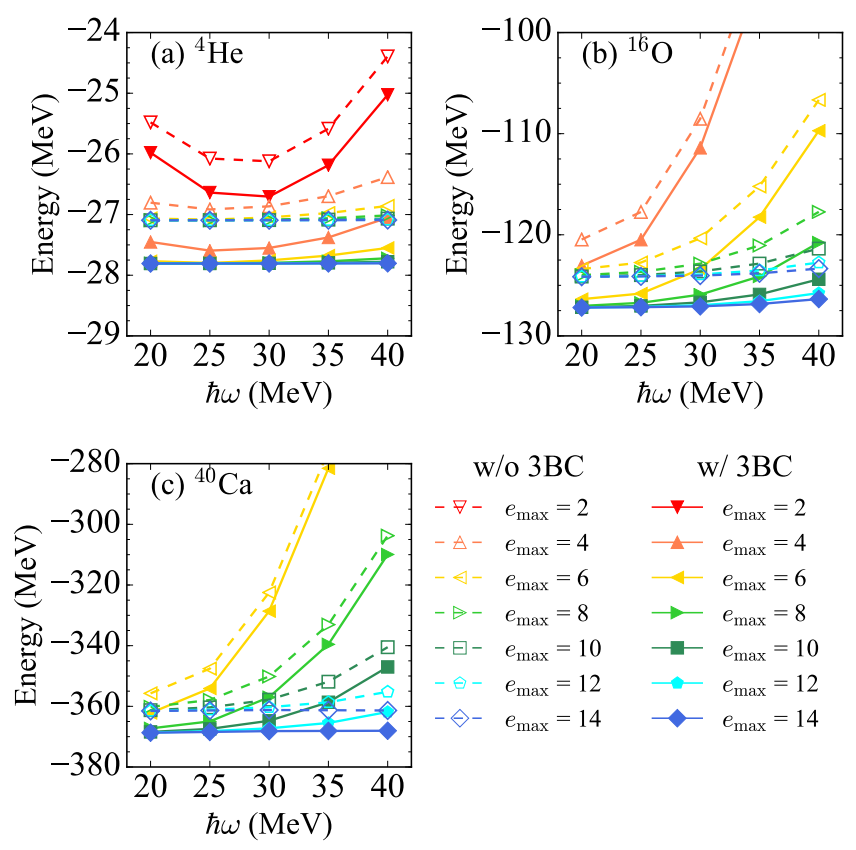

FIG. 1. (color online) Ground-state energies for ${ }^{4} \mathrm{He},{ }^{16} \mathrm{O}$, and ${ }^{40} \mathrm{Ca}$ as functions of $\hbar \omega$ with the $N N+3 N$-full interaction. The dashed (solid) lines calculated without (with) the three-body cluster term energy. The interaction is obtained by SRG evolution of chiral $\mathrm{N}^{3} \mathrm{LO} N N$ [51] and $\mathrm{N}^{2} \mathrm{LO}$ $3 N[6,39]$ interactions up to $\lambda_{\mathrm{SRG}}=1.88 \mathrm{fm}^{-1}$.

energies. In terms of HF-MBPT, the energies $E_{\mathrm{MBPT}}^{1,2 \mathrm{BC}}$, $E_{\mathrm{MBPT}}^{3 \mathrm{BC}}$, and $E_{\text {g.s., MBPT }}$ are evaluated as

$$
\begin{aligned}
E_{\mathrm{MBPT}}^{1,2 \mathrm{BC}} & =E_{\mathrm{HF}}+S_{2}+T_{12}-T_{13}, \\
E_{\mathrm{MBPT}}^{3 \mathrm{BC}} & =2 T_{13}+T_{14}, \\
E_{\text {g.s. }, \mathrm{MBPT}} & =E_{\mathrm{HF}}+S_{2}+T_{12}+T_{13}+T_{14},
\end{aligned}
$$

with the Hartree-Fock energy $E_{\mathrm{HF}}$. Note that $E_{\text {g.s., MBPT }}$ is the third-order HF-MBPT energy. In the figure, the UMOA and HF-MBPT energies are reasonably close to each other and it can be seen that the main contributions of $E^{3 \mathrm{BC}}$ are from the third order hole-hole $\left(T_{13}\right)$ and particle-hole $\left(T_{14}\right)$ ladder diagrams. Also, it is shown that the sum of the higher order terms taken into account in the UMOA is repulsive. Comparing to CCM energies, total UMOA energies (circle) look closer to the CCSD energies (down triangle) than to the $\mathrm{CR}-\mathrm{CC}(2,3)$ energies (pentagon). The $E^{3 \mathrm{BC}}$ are $-0.71,-3.04$, and -7.07 $\mathrm{MeV}$ for ${ }^{4} \mathrm{He},{ }^{16} \mathrm{O}$, and ${ }^{40} \mathrm{Ca}$, respectively, and are only a few percent of the total energies. Since the contributions from four- and higher-body cluster terms are expected to be smaller than those from the three-body cluster term, the UMOA results are converged with respect to the cluster expansion. For ${ }^{16} \mathrm{O}$, our ground-state energy -127.16 $\mathrm{MeV}$ is slightly underbound compared to the experimental energy $(-127.62 \mathrm{MeV})$, while the recent ab initio calculation results show milidly overbound to the experiment, for example, -130.6(1) MeV from in-medium SRG 
TABLE II. Ground-state energies for ${ }^{4} \mathrm{He},{ }^{16} \mathrm{O}$, and ${ }^{40} \mathrm{Ca}$. All the calculation results are obtained at $e_{\max }=14$ and $\hbar \omega=25$ $\mathrm{MeV}$.

\begin{tabular}{clrrr}
\hline \hline \multirow{2}{*}{ Nuclide } & & \multicolumn{2}{c}{$E_{\text {g.s. }(\mathrm{MeV})}$} \\
\cline { 2 - 4 } & $\lambda_{\text {SRG }}\left(\mathrm{fm}^{-1}\right)$ & -27.94 & $N N+3 N-$ ind & $N N+3 N-$ full \\
${ }^{4} \mathrm{He}$ & 1.88 & -27.73 & -25.19 & -27.81 \\
& 2.0 & -27.23 & -25.18 & -27.76 \\
& 2.24 & & -25.16 & -27.62 \\
& & & & -127.16 \\
${ }^{16} \mathrm{O}$ & 1.88 & -167.79 & -119.33 & -126.33 \\
& 2.0 & -162.69 & -119.51 & -124.50 \\
& 2.24 & -152.88 & -119.56 & -127.62 \\
${ }^{40} \mathrm{Ca}$ & 1.88 & -615.62 & -349.08 & -368.44 \\
& 2.0 & -588.45 & -352.03 & -366.14 \\
\hline \hline
\end{tabular}
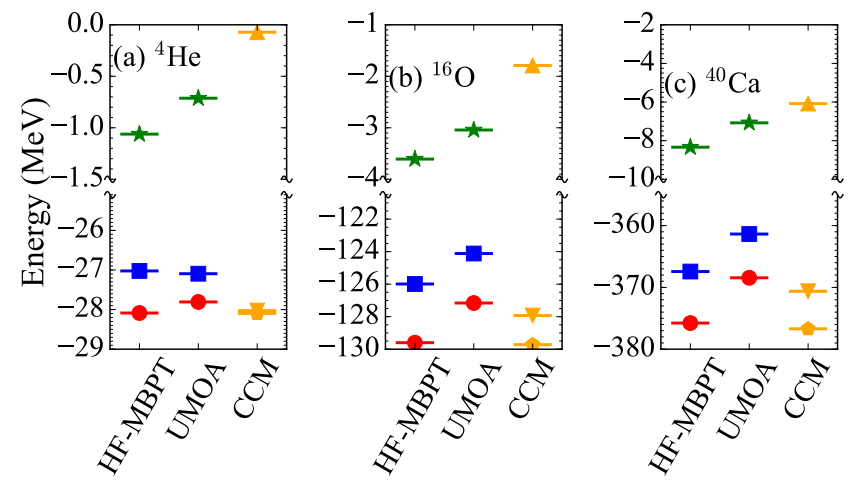

FIG. 2.

(color online) Ground-state energies from the Hartree-Fock basis many-body perturbation theory (HFMBPT), UMOA, and coupled-cluster method (CCM). The energies of HF-MBPT are calculated with Eqs. (39)-(41). The CCM results are taken from Refs. $[18,56]$. The interaction is obtained by SRG evolution of chiral $\mathrm{N}^{3} \mathrm{LO} N N$ [51] and $\mathrm{N}^{2} \mathrm{LO} 3 N[6,39]$ interactions up to $\lambda_{\mathrm{SRG}}=1.88 \mathrm{fm}^{-1}$. For the HF-MBPT and UMOA energies, $E^{1,2 \mathrm{BC}}$ (square), $E^{3 \mathrm{BC}}$ (star), and $E_{\text {g.s. }}$ (circle) are shown. For the CCM energies, CCSD (down triangle), triple correction (up triangle), and CR-CC $(2,3)$ energies (pentagon) are shown.

approach [14] and -129.7 MeV from CCM [18]. Again, this disagreement between our and other $a b$ initio results is same order of the size of the perturbative three-bodycluster contribution and consistent with the accuracy of the UMOA calculations.

As for calculations with $N N$-only and $N N+3 N$-ind interactions, we observe the similar convergence pattern and find the converged results at $e_{\max }=14$ calculations. In Figure 3, the calculated ground-state energies are summarized together with the comparisons to the experimental data. In case of $N N$-only interaction results, as the mass number increases, the ground-state energies show

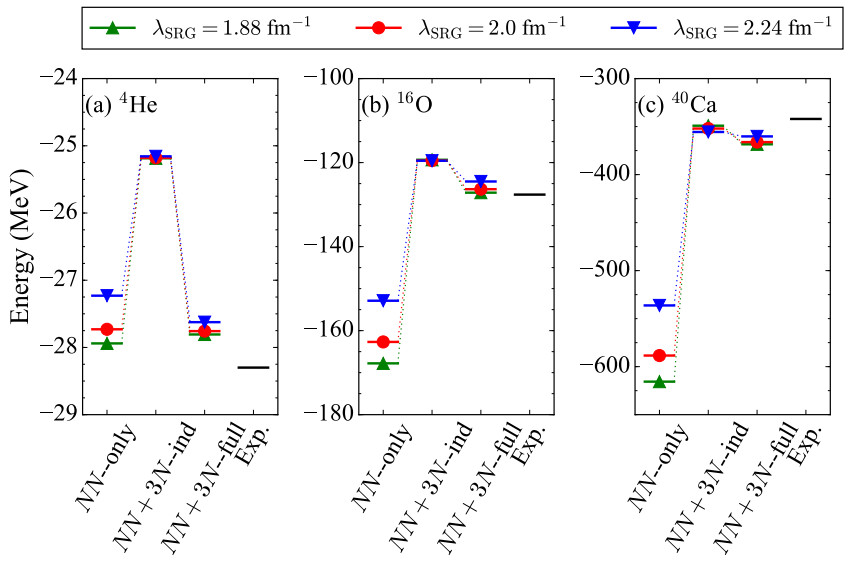

FIG. 3. (color online) Ground-state energies for ${ }^{4} \mathrm{He},{ }^{16} \mathrm{O}$, and ${ }^{40} \mathrm{Ca}$. All the calculation results are obtained at $e_{\max }=$ 14 and $\hbar \omega=25 \mathrm{MeV}$. The experimental data are taken from Ref. [50]

overbinding and $\lambda_{\mathrm{SRG}}$-dependence becomes considerable. By taking the SRG induced $3 N$ interaction into account, the $\lambda_{\mathrm{SRG}}$-dependence is drastically reduced and groundstate energies rise. This $\lambda_{\mathrm{SRG}}$-independence of groundstate energies implies that the contributions from SRG induced four- and many-body interactions are negligible. With the genuine $\chi \mathrm{EFT} \mathrm{N}^{2} \mathrm{LO} 3 N$ interaction, the calculated ground-state energies are comparable to the experimental data for ${ }^{4} \mathrm{He}$ and ${ }^{16} \mathrm{O}$, while overbinding is seen for ${ }^{40} \mathrm{Ca}$. The current choice of the genuine $3 N$ interaction gives $9 \%, 6 \%$, and $4 \%$ attractions for ${ }^{4} \mathrm{He},{ }^{16} \mathrm{O}$, and ${ }^{40} \mathrm{Ca}$, respectively. The energies presented in Fig. 3 are also displayed in Table II. Our ground-state energies show reasonable agreement with the other ab initio results from the same interaction $[14,16,29,39,40,57]$. The explicit treatment of the three-body cluster term seems to be necessary to discuss more precisely the accu- 

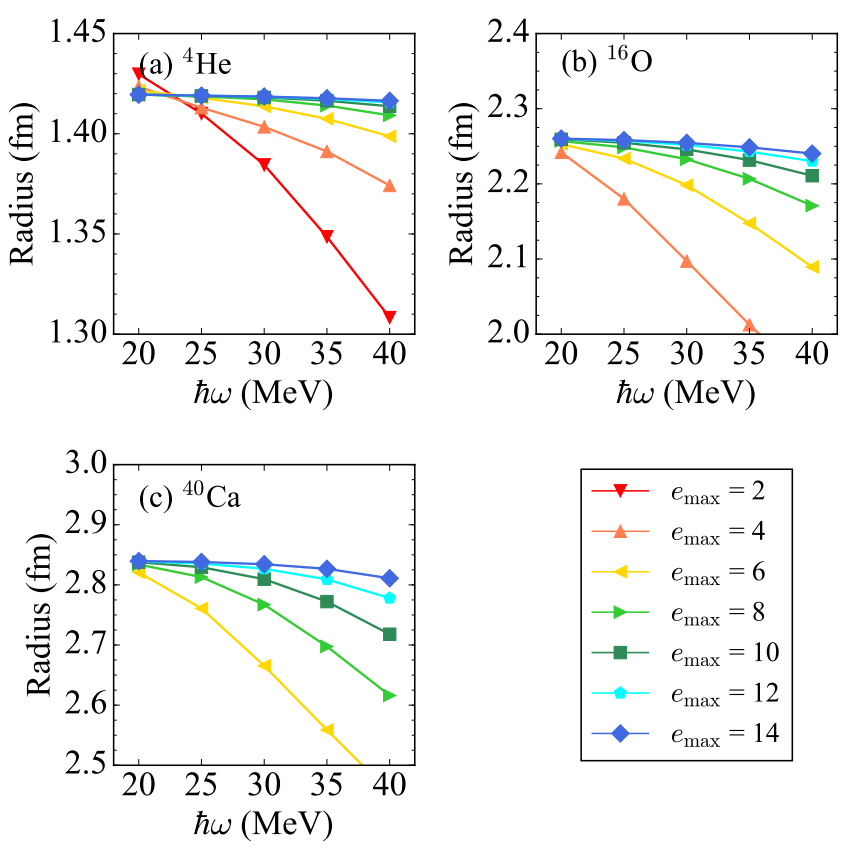

FIG. 4. (color online) Expectation values of bare root-meansquared radius operator for ${ }^{4} \mathrm{He},{ }^{16} \mathrm{O}$, and ${ }^{40} \mathrm{Ca}$ as functions of $\hbar \omega$. Here, $N N+3 N$-full interaction at $\lambda_{\mathrm{SRG}}=1.88 \mathrm{fm}^{-1}$ is employed.

racy of the UMOA calculation. Such works are on going and will be reported in the future publication.

\section{B. Root-mean square radii}

In the same as the ground-state energy calculations, we calculate the expectation values of the bare root-meansquared radius operator with the chiral $N N+3 N$-full interaction at $\lambda_{\mathrm{SRG}}=1.88 \mathrm{fm}^{-1}$ varying both of $\hbar \omega$ and $e_{\max }$ to examine the convergence. The results for ${ }^{4} \mathrm{He},{ }^{16} \mathrm{O}$, and ${ }^{40} \mathrm{Ca}$ are illustrated in Fig. 4. As demonstrated in the figure, calculated radii become $\hbar \omega$ - and $e_{\max }$-independent with increasing $e_{\max }$. At $\hbar \omega=25$ $\mathrm{MeV}$, we find the converged radii within $0.01 \mathrm{fm}$ for all nuclei calculated here. Note that our converged radius of $2.84 \mathrm{fm}$ for ${ }^{40} \mathrm{Ca}$ from the interaction evolved up to $\lambda_{\mathrm{SRG}}=2.0 \mathrm{fm}^{-1}$ shows reasonable agreement with the SCGF result of $2.89 \mathrm{fm}$ [16] from the same interaction.

We also calculate radii for ${ }^{4} \mathrm{He},{ }^{16} \mathrm{O}$, and ${ }^{40} \mathrm{Ca}$ with the $N N$-only and $N N+3 N$-ind interactions in the same manner as with the $N N+3 N$-full interaction. Then, we find converged results at $e_{\max }=14$ and $\hbar \omega=25 \mathrm{MeV}$ within $0.01 \mathrm{fm}$. The results are summarized in Fig. 5 with the comparison to the experimental charge radii [58]. To compare with the experimental charge radii, our charge radii $r_{\text {ch }}$ are evaluated as [59],

$$
r_{\mathrm{ch}}^{2}=r_{\text {g.s. }}^{2}+r_{\mathrm{p}}^{2}+\frac{N}{Z} r_{\mathrm{n}}^{2}+\frac{3}{4 m^{2}} \text {. }
$$

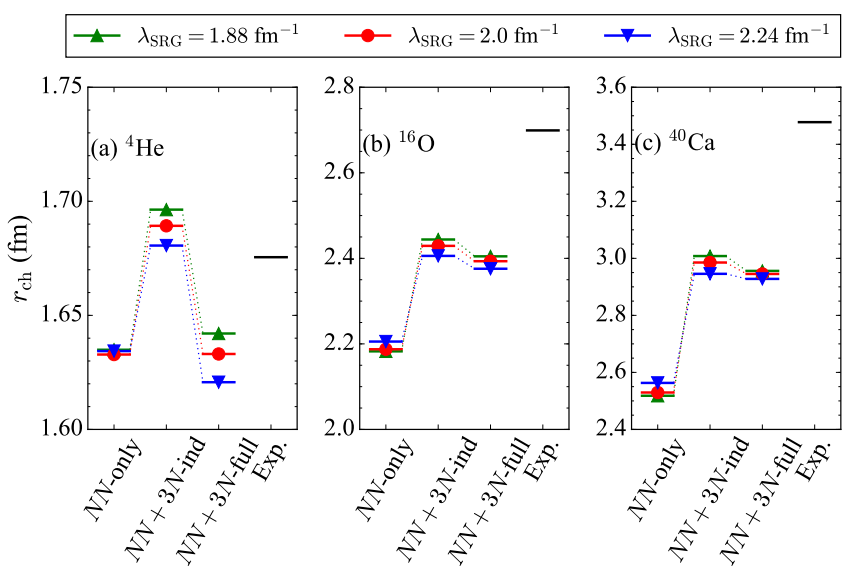

FIG. 5. (color online) Charge radii for ${ }^{4} \mathrm{He},{ }^{16} \mathrm{O}$, and ${ }^{40} \mathrm{Ca}$. All calculation results are obtained at $\hbar \omega=25 \mathrm{MeV}$ and $e_{\max }=14$. To evaluate the charge radii, the bare meansquared radius operators are used. The experimental data are taken from Ref. [58].

Here, we use $r_{\mathrm{p}}=0.8751(61) \mathrm{fm}[60], r_{\mathrm{n}}^{2}=-0.1161(22)$ $\mathrm{fm}^{2}[60]$, and $3 / 4 \mathrm{~m}^{2}=0.033 \mathrm{fm}^{2}$, with the averaged nucleon mass $m=938.919 \mathrm{MeV} / c^{2}$. Note that we assume the equivalence of point-proton and point-nucleon distributions in Eq. (42). This assumption would be reasonable because our targets are $N=Z$ stable nuclei. In Fig. 6, the charge radii from $N N$-only interactions are obviously smaller than experimental data, especially for ${ }^{16} \mathrm{O}$ and ${ }^{40} \mathrm{Ca}$ and consistent with overbinding groundstate energies from those. The SRG induced three-body operator contributes to spread the nuclear distribution out. Then, the $\lambda_{\mathrm{SRG}}$-dependence is slightly enhanced. With this particular Hamiltonian, the genuine $3 N$ interaction shrinks nuclei and the calculated radii are clearly smaller than the experimental data.

As a possible reason for the calculated small radii, for example, the nuclear interaction can be considered. In fact, the simultaneous reproduction of ground-state energies and radii were accomplished with the $\chi \mathrm{EFT} \mathrm{N}^{2} \mathrm{LO}$ $N N+3 N$ interaction fitted by using some selected data of nuclei up to $A=25$ [61]. In addition, the saturation property of infinite nuclear matter was reproduced with the combinations of the softened $\mathrm{N}^{3} \mathrm{LO} N N$ and bare $\mathrm{N}^{2} \mathrm{LO} 3 \mathrm{~N}$ interactions whose low-energy constants are fitted to reproduce data of the few body systems [19]. The simultaneous reproduction of ground-state energies and radii for finite nuclei with such interactions were compensated by the recent $a b$ initio calculations [62]. As another possibility, we can consider amending the treatment of radius operator. In earlier no-core shell model (NCSM) studies [36, 37], the effect of the SRG evolution to several operators were investigated for few-body systems. However, such effects for medium-mass nuclei have not been clarified yet. In this work, we investigate the effect of the SRG evolution to the radius operator.

We calculate the expectation value with the bare, two- 
TABLE III. Root-mean-squared radii $r_{\text {g.s. }}$ for ${ }^{4} \mathrm{He},{ }^{16} \mathrm{O}$, and ${ }^{40} \mathrm{Ca}$ calculated with the bare, two-body evolved (2B), and threebody evolved (3B) radius operators. The results from both $N N+3 N$-ind and $N N+3 N$-full are displayed. All the calculation results are obtained at $e_{\max }=14$ and $\hbar \omega=25 \mathrm{MeV}$.

\begin{tabular}{|c|c|c|c|c|c|c|c|}
\hline \multirow[b]{3}{*}{ Nuclide } & \multirow[b]{3}{*}{$\lambda_{\mathrm{SRG}}\left(\mathrm{fm}^{-1}\right)$} & \multicolumn{6}{|c|}{$r_{\text {g.s. }}(\mathrm{fm})$} \\
\hline & & \multicolumn{3}{|c|}{$N N+3 N-$ ind } & \multicolumn{3}{|c|}{$N N+3 N-$ full } \\
\hline & & Bare & $2 \mathrm{~B}$ & $3 \mathrm{~B}$ & Bare & $2 \mathrm{~B}$ & $3 \mathrm{~B}$ \\
\hline \multirow{3}{*}{${ }^{4} \mathrm{He}$} & 1.88 & 1.48 & 1.47 & 1.46 & 1.42 & 1.40 & 1.39 \\
\hline & 2.0 & 1.47 & 1.46 & 1.45 & 1.41 & 1.40 & 1.39 \\
\hline & 2.24 & 1.46 & 1.45 & 1.45 & 1.39 & 1.39 & 1.38 \\
\hline \multirow{3}{*}{${ }^{16} \mathrm{O}$} & 1.88 & 2.32 & 2.29 & 2.26 & 2.28 & 2.24 & 2.22 \\
\hline & 2.0 & 2.30 & 2.27 & 2.25 & 2.27 & 2.23 & 2.21 \\
\hline & 2.24 & 2.28 & 2.25 & 2.24 & 2.25 & 2.22 & 2.20 \\
\hline \multirow{3}{*}{${ }^{40} \mathrm{Ca}$} & 1.88 & 2.91 & 2.89 & 2.85 & 2.86 & 2.83 & 2.79 \\
\hline & 2.0 & 2.89 & 2.86 & 2.83 & 2.84 & 2.82 & 2.79 \\
\hline & 2.24 & 2.84 & 2.82 & 2.80 & 2.83 & 2.80 & 2.78 \\
\hline
\end{tabular}

body evolved, and three-body evolved mean-squared radius operators. In the same as the bare radius operator cases, we check the convergence pattern and find the root-mean-squared radius results converged within 0.01 fm. Evaluated charge radii are illustrated in Fig. 6. Corresponding to Fig. 6, final results for root-mean-squared radii from $N N+3 N$-ind and $N N+3 N$-full interactions are exhibited in Table III. For all nuclei, as we calculate with higher-body evolved operator, the radii tend to be small and go opposite direction to the data. This behavior is consistent with the earlier NCSM results [36]. Also, similar to the role of the SRG induced three-body interaction, consistently evolved operator moderately reduces the $\lambda_{\mathrm{SRG}}$-dependence of radii. Therefore, it can be concluded that the consistent SRG evolution of the radius operator does not give the significant change compared to the experimental data. This is consistent with the longrange nature of the radius operator [36]. There are some insights about the effect of consistent SRG evolution of radius operator [63]. In this work, however, we do not observe the enhancement of radii discussed in Ref. [63]. The quantitative reproduction of nuclear size is still an open question.

Finally, let us see the saturation plot of the groundstate energies and radii from this work and from other calculations. In Figure 7, the ground-state energies per nucleon and charge radii for ${ }^{4} \mathrm{He},{ }^{16} \mathrm{O}$, and ${ }^{40} \mathrm{Ca}$ with various nuclear interactions are plotted. In the figure, the results obtained in the present work are shown by solid symbols. The triangles, squares, and circles are the results with the $N N$-only, $N N+3 N$-ind, and $N N+3 N$-full interactions, respectively. For visibility, only the results at $\lambda_{\mathrm{SRG}}=2.0 \mathrm{fm}^{-1}$ are marked in the figure. The open symbols are from other calculations $[61,62,64,65]$. The experimental data are indicated by thick crosses $[50,58]$. The results with the
$N N$ interactions, $N N$-only (triangle), $N N+3 N$-ind (square), CD-Bonn (diamond), and AV18 (left triangle), fail to reproduce the experimental data. The inclusion of $3 \mathrm{~N}$ interactions, $N N+3 \mathrm{~N}$ full (circle) and $\mathrm{AV}+\mathrm{UIX}$ (right triangle), does not help the calculated radius come close to the experimental data for ${ }^{16} \mathrm{O}$ and ${ }^{40} \mathrm{Ca}$. On the other hand, the other type of chiral $N N+3 N$ interactions (pentagon and hexagon) show nice agreement with the data. As seen in the figure, calculation results are scattered even if the $N N$ and $3 N$ interactions are used, and further investigations are indispensable how both of the $N N$ and $3 N$ interactions can be determined.

\section{CONCLUSION}

In the present work, we have calculated the groundstate energies and radii for ${ }^{4} \mathrm{He},{ }^{16} \mathrm{O}$, and ${ }^{40} \mathrm{Ca}$ with the UMOA from $N N$ and $3 N$ interactions based on the $\chi \mathrm{EFT}$ for the first time. To obtain the computationally tractable Hamiltonian in the UMOA, we employed the SRG evolution and the NO2B approximation.

The resulting ground-state energies and radii agree with the recent $a b$ initio calculation results within a few percent. To discuss the accuracy of the UMOA calculation more precisely, we are going to extend the UMOA framework and directly treat the three-body cluster term beyond the NO2B approximation. The results will be discussed in the future publication.

In addition to expectation values for the bare radius operator, in the present work, we have evaluated those for the two- and three-body SRG evolved radius operators. By taking higher-body evolved operator into account, calculated radii slightly shrink, while the $\lambda_{\mathrm{SRG}^{-}}$ dependence of radii is reduced as we keep up to threebody terms. Therefore, it is unlikely to reproduce the 


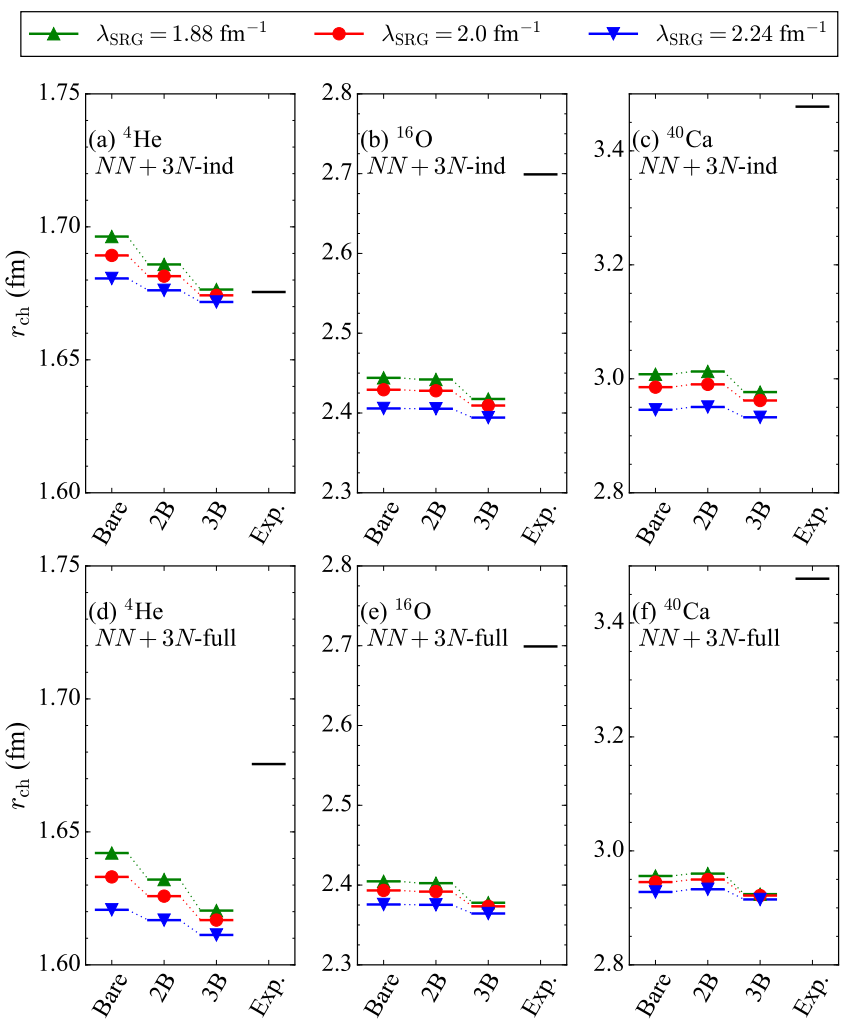

FIG. 6. (color online) Charge radii evaluated with the expectation values of bare, two-body (2B) SRG evolved, and three-body (3B) SRG evolved mean-squared radius operator for ${ }^{4} \mathrm{He}[(\mathrm{a})$ and $(\mathrm{d})],{ }^{16} \mathrm{O}\left[(\mathrm{b})\right.$ and (e)], and ${ }^{40} \mathrm{Ca}[(\mathrm{c})$ and (f)]. All calculation results are obtained at $\hbar \omega=25 \mathrm{MeV}$ and $e_{\max }=14$. The calculation results in panels (a), (b), and (c) are calculated with $N N+3 N$-ind interactions and the calculation results in panels (d), (e), and (f) are calculated with $N N+3 N$-full interactions.

nuclear radii with the interactions employed in this work, even if we continue to include many-body terms induced by SRG evolution. The simultaneous reproduction of the ground-state energies and radii strongly depend on empolyed nuclear interactions. To specify the proper choice of nuclear interactions, further investigations are needed.

\section{ACKNOWLEDGMENTS}

We thank R. Roth for providing us the coupled-cluster calculation results. This work was supported in part by
JSPS KAKENHI Grant No. JP16J05707 and by the Program for Leading Graduate Schools, MEXT, Japan. This work was also supported in part by MEXT as "Priority Issue on post-K computer" (Elucidation of the Fundamental Laws and Evolution of the Universe) and JICFuS (Projects No. hp160211 and No. hp170230) and CNSRIKEN joint project for large-scale nuclear structure calculations, and the NSERC Grant No. SAPIN-201600033. TRIUMF receives federal funding via a contri-

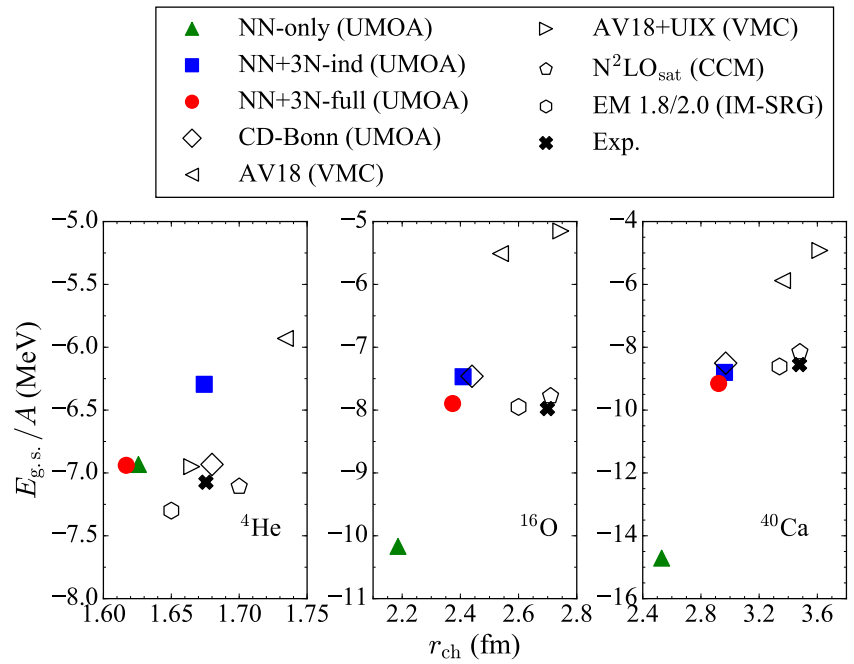

FIG. 7. (color online) Ground-state energies per nucleon for ${ }^{4} \mathrm{He},{ }^{16} \mathrm{O}$, and ${ }^{40} \mathrm{Ca}$ as a function of $r_{\mathrm{ch}}$. The triangle, square, circle symbols are UMOA results from $N N$-only, $N N+3 N$-ind, and $N N+3 N$-full interactions evolved up to $2.0 \mathrm{fm}^{-1}$, respectively. The diamonds are taken from earlier UMOA results with the CD-Bonn interaction [64]. The left and right triangles are variational Monte Carlo calculations (VMC) with the AV18 and AV18+UIX interactions, respectively [65]. The pentagons and hexagons are taken from the coupled-cluster method (CCM) [61] and in-medium SRG (IMSRG) [62] results, respectively. Thick cross symbols indicate the experimental data $[50,58]$.

bution agreement with the National Research Council of Canada. A part of numerical calculations were done on Oakforest-PACS, Supercomputing Division, Information Technology Center, the University of Tokyo.
[1] E. Epelbaum, H.-W. Hammer, and U.-G. Meißner, Rev. Mod. Phys. 81, 1773 (2008), arXiv:0811.1338.

[2] R. Machleidt and D. Entem, Phys. Rep. 503, 1 (2011), arXiv:1105.2919.

[3] S. Binder, A. Calci, E. Epelbaum, R. J. Furnstahl, J. Golak, K. Hebeler, H. Kamada, H. Krebs, J. Langhammer,
S. Liebig, P. Maris, U.-G. Meißner, D. Minossi, A. Nogga, H. Potter, R. Roth, R. Skibiński, K. Topolnicki, J. P. Vary, and H. Witała, Phys. Rev. C 93, 044002 (2016), arXiv:1505.07218.

[4] D. R. Entem, R. Machleidt, and Y. Nosyk, Phys. Rev. C 96, 024004 (2017), arXiv:1703.05454. 
[5] S. Binder, A. Calci, E. Epelbaum, R. J. Furnstahl, J. Golak, K. Hebeler, T. Hüther, H. Kamada, H. Krebs, P. Maris, U.-G. Meißner, A. Nogga, R. Roth, R. Skibiński, K. Topolnicki, J. P. Vary, K. Vobig, and H. Witała, Phys. Rev. C 98, 014002 (2018), arXiv:1807.02848.

[6] P. Navratil, V. G. Gueorguiev, J. P. Vary, W. E. Ormand, and A. Nogga, Phys. Rev. Lett. 99, 042501 (2007), arXiv:0701038 [nucl-th].

[7] G. Hupin, S. Quaglioni, J. Langhammer, P. Navrátil, A. Calci, and R. Roth, Few-Body Syst. 55, 1013 (2014), arXiv:1401.0365.

[8] J. Langhammer, P. Navrátil, S. Quaglioni, G. Hupin, A. Calci, and R. Roth, Phys. Rev. C 91, 021301 (2015), arXiv:1411.2541.

[9] G. Hupin, S. Quaglioni, and P. Navrátil, Phys. Rev. Lett. 114, 212502 (2014), arXiv:1412.4101.

[10] T. Otsuka, T. Suzuki, J. D. Holt, A. Schwenk, and Y. Akaishi, Phys. Rev. Lett. 105, 032501 (2009), arXiv:0908.2607.

[11] G. Hagen, M. Hjorth-Jensen, G. R. Jansen, R. Machleidt, and T. Papenbrock, Phys. Rev. Lett. 108, 242501 (2012), arXiv:1202.2839.

[12] G. Hagen, M. Hjorth-Jensen, G. R. Jansen, R. Machleidt, and T. Papenbrock, Phys. Rev. Lett. 109, 032502 (2012), arXiv: 1204.3612

[13] H. Hergert, S. Binder, A. Calci, J. Langhammer, and R. Roth, Phys. Rev. Lett. 110, 242501 (2013), arXiv:1302.7294.

[14] H. Hergert, S. K. Bogner, S. Binder, A. Calci, J. Langhammer, R. Roth, and A. Schwenk, Phys. Rev. C 87, 034307 (2013), arXiv:1212.1190.

[15] H. Hergert, S. K. Bogner, T. D. Morris, S. Binder, A. Calci, J. Langhammer, and R. Roth, Phys. Rev. C 90, 041302 (2014), arXiv:1408.6555.

[16] V. Somà, A. Cipollone, C. Barbieri, P. Navrátil, and T. Duguet, Phys. Rev. C 89, 061301 (2014), arXiv:1312.2068.

[17] A. Cipollone, C. Barbieri, and P. Navrátil, Phys. Rev. C 92, 014306 (2015), arXiv:1412.0491.

[18] S. Binder, J. Langhammer, A. Calci, and R. Roth, Phys. Lett. B 736, 119 (2014), arXiv:1312.5685.

[19] K. Hebeler, S. K. Bogner, R. J. Furnstahl, A. Nogga, and A. Schwenk, Phys. Rev. C 83, 031301 (2011), arXiv:1012.3381.

[20] M. Kohno, Phys. Rev. C 88, 064005 (2013) arXiv:1309.4556, Phys. Rev. C 96, 059903(E) (2017).

[21] G. Hagen, T. Papenbrock, A. Ekström, K. A. Wendt, G. Baardsen, S. Gandolfi, M. Hjorth-Jensen, and C. J. Horowitz, Phys. Rev. C 89, 014319 (2014), arXiv:1311.2925.

[22] I. Tews, S. Gandolfi, A. Gezerlis, and A. Schwenk, Phys. Rev. C 93, 024305 (2016), arXiv:1507.05561.

[23] B. R. Barrett, P. Navrátil, and J. P. Vary, Prog. Part. Nucl. Phys. 69, 131 (2013).

[24] J. Carlson, S. Gandolfi, F. Pederiva, S. C. Pieper, R. Schiavilla, K. E. Schmidt, and R. B. Wiringa, Rev. Mod. Phys. 87, 1067 (2015), arXiv:1412.3081.

[25] D. Lee, Prog. Part. Nucl. Phys. 63, 117 (2009), arXiv:0804.3501.

[26] G. Hagen, T. Papenbrock, M. Hjorth-Jensen, and D. J. Dean, Reports Prog. Phys. 77, 096302 (2014), arXiv:1312.7872.

[27] W. Dickhoff and C. Barbieri, Prog. Part. Nucl. Phys. 52,
377 (2004), arXiv:0402034 [nucl-th].

[28] H. Hergert, S. Bogner, T. Morris, A. Schwenk, and K. Tsukiyama, Phys. Rep. 621, 165 (2016), arXiv:1512.06956.

[29] A. Tichai, J. Langhammer, S. Binder, and R. Roth, Phys. Lett. B 756, 283 (2016), arXiv:1601.03703.

[30] B. S. Hu, F. R. Xu, Z. H. Sun, J. P. Vary, and T. Li, Phys. Rev. C 94, 014303 (2016), arXiv:1604.07146.

[31] T. D. Morris, J. Simonis, S. R. Stroberg, C. Stumpf, G. Hagen, J. D. Holt, G. R. Jansen, T. Papenbrock, R. Roth, and A. Schwenk, Phys. Rev. Lett. 120, 152503 (2018), arXiv:1709.02786.

[32] K. Suzuki and R. Okamoto, Prog. Theor. Phys. 92, 1045 (1994).

[33] T. Miyagi, T. Abe, R. Okamoto, and T. Otsuka, Phys. Rev. C 96, 054312 (2017), arXiv:1708.00177.

[34] S. K. Bogner, R. J. Furnstahl, and R. J. Perry, Phys. Rev. C 75, 061001 (2007), arXiv:0611045 [nucl-th].

[35] V. Lapoux, V. Somà, C. Barbieri, H. Hergert, J. D. Holt, and S. R. Stroberg, Phys. Rev. Lett. 117, 052501 (2016), arXiv:1605.07885.

[36] M. D. Schuster, S. Quaglioni, C. W. Johnson, E. D. Jurgenson, and P. Navrátil, Phys. Rev. C 90, 011301 (2014), arXiv:1402.7106.

[37] M. D. Schuster, S. Quaglioni, C. W. Johnson, E. D. Jurgenson, and P. Navrátil, Phys. Rev. C 92, 014320 (2015), arXiv:1304.5491.

[38] E. D. Jurgenson, P. Navrátil, and R. J. Furnstahl, Phys. Rev. Lett. 103, 082501 (2009), arXiv:0905.1873.

[39] R. Roth, S. Binder, K. Vobig, A. Calci, J. Langhammer, and P. Navrátil, Phys. Rev. Lett. 109, 052501 (2012), arXiv:1112.0287.

[40] S. Binder, J. Langhammer, A. Calci, P. Navrátil, and R. Roth, Phys. Rev. C 87, 021303 (2013), arXiv:1211.4748.

[41] I. Shavitt and R. J. Bartlett, Many - Body Methods Chem. Phys. MBPT Coupled-Cluster Theory (Cambridge University Press, Cambridge, 2009).

[42] K. Suzuki, R. Okamoto, and H. Kumagai, Phys. Rev. C 36, 804 (1987).

[43] K. Suzuki, Prog. Theor. Phys. 79, 330 (1988).

[44] S. Ôkubo, Prog. Theor. Phys. 12, 603 (1954).

[45] S. Lee and K. Suzuki, Phys. Lett. B 91, 173 (1980).

[46] K. Suzuki and S. Y. Lee, Prog. Theor. Phys. 64, 2091 (1980).

[47] K. Suzuki and R. Okamoto, Prog. Theor. Phys. 70, 439 (1983).

[48] K. Suzuki and R. Okamoto, Prog. Theor. Phys. 72, 534 (1984).

[49] K. Suzuki and R. Okamoto, Prog. Theor. Phys. 76, 127 (1986).

[50] M. Wang, G. Audi, A. Wapstra, F. Kondev, M. MacCormick, X. Xu, and B. Pfeiffer, Chinese Phys. C 36, 1603 (2012).

[51] D. R. Entem and R. Machleidt, Phys. Rev. C 68, 041001 (2003), arXiv:0304018 [nucl-th].

[52] P. Navrátil, Few-Body Syst. 41, 117 (2007), arXiv:0707.4680.

[53] R. Roth, A. Calci, J. Langhammer, and S. Binder, Phys. Rev. C 90, 024325 (2014), arXiv:1311.3563.

[54] D. Gazit, S. Quaglioni, and P. Navrátil, Phys. Rev. Lett. 103, 102502 (2009), arXiv:0812.4444, Phys. Rev. Lett. 122, 029901(E) (2019). 
[55] L. E. Marcucci, A. Kievsky, S. Rosati, R. Schiavilla, and M. Viviani, Phys. Rev. Lett. 108, 052502 (2012), arXiv:1109.5563, Phys. Rev. Lett. 121, 049901(E) (2018).

[56] S. Binder, P. Piecuch, A. Calci, J. Langhammer, P. Navrátil, and R. Roth, Phys. Rev. C 88, 054319 (2013), arXiv:1309.1123.

[57] A. Cipollone, C. Barbieri, and P. Navrátil, Phys. Rev. Lett. 111, 062501 (2013), arXiv:1303.4900v1.

[58] I. Angeli and K. Marinova, At. Data Nucl. Data Tables 99, 69 (2013).

[59] J. L. Friar and J. W. Negele, Adv. Nucl. Phys. 8, 219 (1975).

[60] C. Patrignani, K. Agashe, G. Aielli, C. Amsler, M. Antonelli, D. M. Asner, H. Baer, S. Banerjee, R. M. Barnett, T. Basaglia, C. W. Bauer, J. J. Beatty, V. I. Belousov, J. Beringer, S. Bethke, H. Bichsel, O. Biebel, E. Blucher, G. Brooijmans, O. Buchmueller, V. Burkert, M. A. Bychkov, R. N. Cahn, M. Carena, A. Ceccucci, A. Cerri, D. Chakraborty, M. C. Chen, R. S. Chivukula, K. Copic, G. Cowan, O. Dahl, G. D'Ambrosio, T. Damour, D. De Florian, A. De Gouvêa, T. DeGrand, P. De Jong, G. Dissertori, B. A. Dobrescu, M. D'Onofrio, M. Doser, M. Drees, H. K. Dreiner, D. A. Dwyer, P. Eerola, S. Eidelman, J. Ellis, J. Erler, V. V. Ezhela, W. Fetscher, B. D. Fields, B. Foster, A. Freitas, H. Gallagher, L. Garren, H. J. Gerber, G. Gerbier, T. Gershon, T. Gherghetta, A. A. Godizov, M. Goodman, C. Grab, A. V. Gritsan, C. Grojean, D. E. Groom, M. Grünewald, A. Gurtu, T. Gutsche, H. E. Haber, K. Hagiwara, C. Hanhart, S. Hashimoto, Y. Hayato, K. G. Hayes, A. Hebecker, B. Heltsley, J. J. Hernández-Rey, K. Hikasa, J. Hisano, A. Höcker, J. Holder, A. Holtkamp, J. Huston, T. Hyodo, K. Irwin, J. D. Jackson, K. F. Johnson, M. Kado, M. Karliner, U. F. Katz, S. R. Klein, E. Klempt, R. V. Kowalewski, F. Krauss, M. Kreps, B. Krusche, Y. V. Kuyanov, Y. Kwon, O. Lahav, J. Laiho, P. Langacker, A. Liddle, Z. Ligeti, C. J. Lin, C. Lippmann, T. M. Liss, L. Littenberg, K. S. Lugovsky, S. B. Lugovsky, A. Lusiani, Y. Makida, F. Maltoni, T. Mannel, A. V. Manohar, W. J. Marciano, A. D. Martin, A. Masoni, J. Matthews,
U. G. Meißner, D. Milstead, R. E. Mitchell, P. Molaro, K. Mönig, F. Moortgat, M. J. Mortonson, H. Murayama, K. Nakamura, M. Narain, P. Nason, S. Navas, M. Neubert, P. Nevski, Y. Nir, K. A. Olive, S. Pagan Griso, J. Parsons, J. A. Peacock, M. Pennington, S. T. Petcov, V. A. Petrov, A. Piepke, A. Pomarol, A. Quadt, S. Raby, J. Rademacker, G. Raffelt, B. N. Ratcliff, P. Richardson, A. Ringwald, S. Roesler, S. Rolli, A. Romaniouk, L. J. Rosenberg, J. L. Rosner, G. Rybka, R. A. Ryutin, C. T. Sachrajda, Y. Sakai, G. P. Salam, S. Sarkar, F. Sauli, O. Schneider, K. Scholberg, A. J. Schwartz, D. Scott, V. Sharma, S. R. Sharpe, T. Shutt, M. Silari, T. Sjöstrand, P. Skands, T. Skwarnicki, J. G. Smith, G. F. Smoot, S. Spanier, H. Spieler, C. Spiering, A. Stahl, S. L. Stone, Y. Sumino, T. Sumiyoshi, M. J. Syphers, F. Takahashi, M. Tanabashi, K. Terashi, J. Terning, R. S. Thorne, L. Tiator, M. Titov, N. P. Tkachenko, N. A. Törnqvist, D. Tovey, G. Valencia, R. Van De Water, N. Varelas, G. Venanzoni, M. G. Vincter, P. Vogel, A. Vogt, S. P. Wakely, W. Walkowiak, C. W. Walter, D. Wands, D. R. Ward, M. O. Wascko, G. Weiglein, D. H. Weinberg, E. J. Weinberg, M. White, L. R. Wiencke, S. Willocq, C. G. Wohl, L. Wolfenstein, J. Womersley, C. L. Woody, R. L. Workman, W. M. Yao, G. P. Zeller, O. V. Zenin, R. Y. Zhu, F. Zimmermann, P. A. Zyla, J. Anderson, G. Harper, V. S. Lugovsky, and P. Schaffner, Chinese Phys. C 40, 100001 (2016).

[61] A. Ekström, G. R. Jansen, K. A. Wendt, G. Hagen, T. Papenbrock, B. D. Carlsson, C. Forssén, M. HjorthJensen, P. Navrátil, and W. Nazarewicz, Phys. Rev. C 91, 051301 (2015), arXiv:1502.04682.

[62] J. Simonis, S. R. Stroberg, K. Hebeler, J. D. Holt, and A. Schwenk, Phys. Rev. C 96, 014303 (2017), arXiv:1704.02915.

[63] G. A. Miller, A. Beck, S. M.-T. Beck, L. B. Weinstein, E. Piasetzky, and O. Hen, arXiv:1805.12099.

[64] T. Miyagi, T. Abe, R. Okamoto, and T. Otsuka, Prog. Theor. Exp. Phys. 2015, $041 D 01$ (2015), arXiv: 1504.04965.

[65] D. Lonardoni, A. Lovato, S. C. Pieper, and R. B. Wiringa, Phys. Rev. C 96, 024326 (2017), arXiv:1705.04337. 